\author{
Klaudyna Grzelakowska ${ }^{1}$, Michał Kasprzak², Jacek Kryś3 \\ ${ }^{1}$ Faculty of Medicine, Nicolaus Copernicus University in Torun, Ludwik Rydygier Collegium Medicum in Bydgoszcz, Poland \\ 2Department of Cardiology and Internal Medicine, Nicolaus Copernicus University in Torun, Ludwik Rydygier Collegium Medicum \\ in Bydgoszcz, Poland \\ ${ }^{3}$ Antoni Jurasz University Hospital No. 1, Nicolaus Copernicus University in Torun, Ludwik Rydygier Collegium Medicum in Bydgoszcz, Poland
}

\title{
COVID-19 and diabetes: a deadly duo?
}

\author{
Corresponding author: \\ Klaudyna Grzelakowska, Faculty \\ of Medicine, Nicolaus Copernicus \\ University in Torun, Ludwik Rydygier \\ Collegium Medicum in Bydgoszcz, \\ Poland, \\ e-mail: klaudyna.grzelakowska@gmail.com
}

Medical Research Journal 2021

Volume 6, Number 2, 119-124

DOI: 10.5603/MRJ.2021.0030

Copyright (c) 2021 Via Medica

ISSN 2451-2591

e-ISSN 2451-4101

\begin{abstract}
Introduction: Among the patients with severe or fatal COVID-19 a high prevalence of comorbidities is noted, diabetes being one of them. The objective of the study was to analyse the relation of COVID-19 and diabetes in respect of their influence on hospitalization's length and outcome.

Materials and methods: The data acquired from the database of Antoni Jurasz University Hospital No. 1 in Bydgoszcz were analysed. The analysis included 1,051 adult COVID-19 positive patients that were hospitalized between March 14, 2020, and April 12, 2021. The collected data included a history of diabetes with differentiation between type 1 and type 2, hospitalization outcome (discharge or death), length of hospital stay.

Results: The prevalence of diabetes in the study group was determined to be $2.09 \%$ and $10.18 \%$ for diabetes type 1 and 2, respectively, totalling 12.27\% ( $n=129)$. Most of the patients (87.54\%) have been discharged while $12.46 \%$ have died. The diabetic patients accounted for $11.63 \%$ of the discharges and $16.79 \%$ of deaths. The mortality rates in the group of insulin-dependent diabetes were the highest, namely $27.27 \%$ vs. $12.15 \%$ in the reaming study population $(p=0.0720)$. Hospitalization's length did not differ according to diabetes occurrence as on average it amounted to 15.90 days in diabetic patients and 14.44 days in non-diabetic ones.

Conclusions: COVID-19 and type 1 diabetes may constitute a deadly duo. Further studies that include patients with insulin-dependent diabetes are needed to better understand the impact of diabetes and COVID-19 on mortality and hospitalization's length.

Key words: SARS-CoV-2, coronavirus, mortality, length of stay, insulin-dependent, type 1 diabetes, type 2 diabetes
\end{abstract}

Med Res J 2021; 6 (2): 119-124

\section{Introduction}

Nowadays, in the era of coronavirus disease 2019 (COVID-19) global scientific efforts are centred around fighting and understanding the pandemic. More and more research are focused on the impact of concomitant diseases on the outcome and the course of the infection caused by severe acute respiratory syndrome coronavirus 2 (SARS-CoV-2). The influence of comorbidities on the severity of COVID-19 cannot be overstated. Among the patients presenting with severe illness and those who died due to the SARS-CoV-2 infection a high prevalence of concomitant diseases is noted, diabetes being one of them [1].
With a global prevalence of 463 million, diabetes is one of the most common chronic conditions worldwide [2]. Hence the diabetic population represents a large vulnerable group of COVID-19 positive patients. Diabetes, especially the most common non-insulin-dependent variant, diabetes type 2 , is perceived as a risk factor for a poor outcome in patients with COVID-19 [3]. The proposed reasons for worse prognosis in diabetic patients include but are not limited to their specific characteristics, namely age, sex, and ethnicity, as well as other comorbid conditions including hypertension and obesity [1]. On that account, whether diabetes itself contributes to the infection remains controversial [4]. Nevertheless, possible pathophysiological mechanisms 


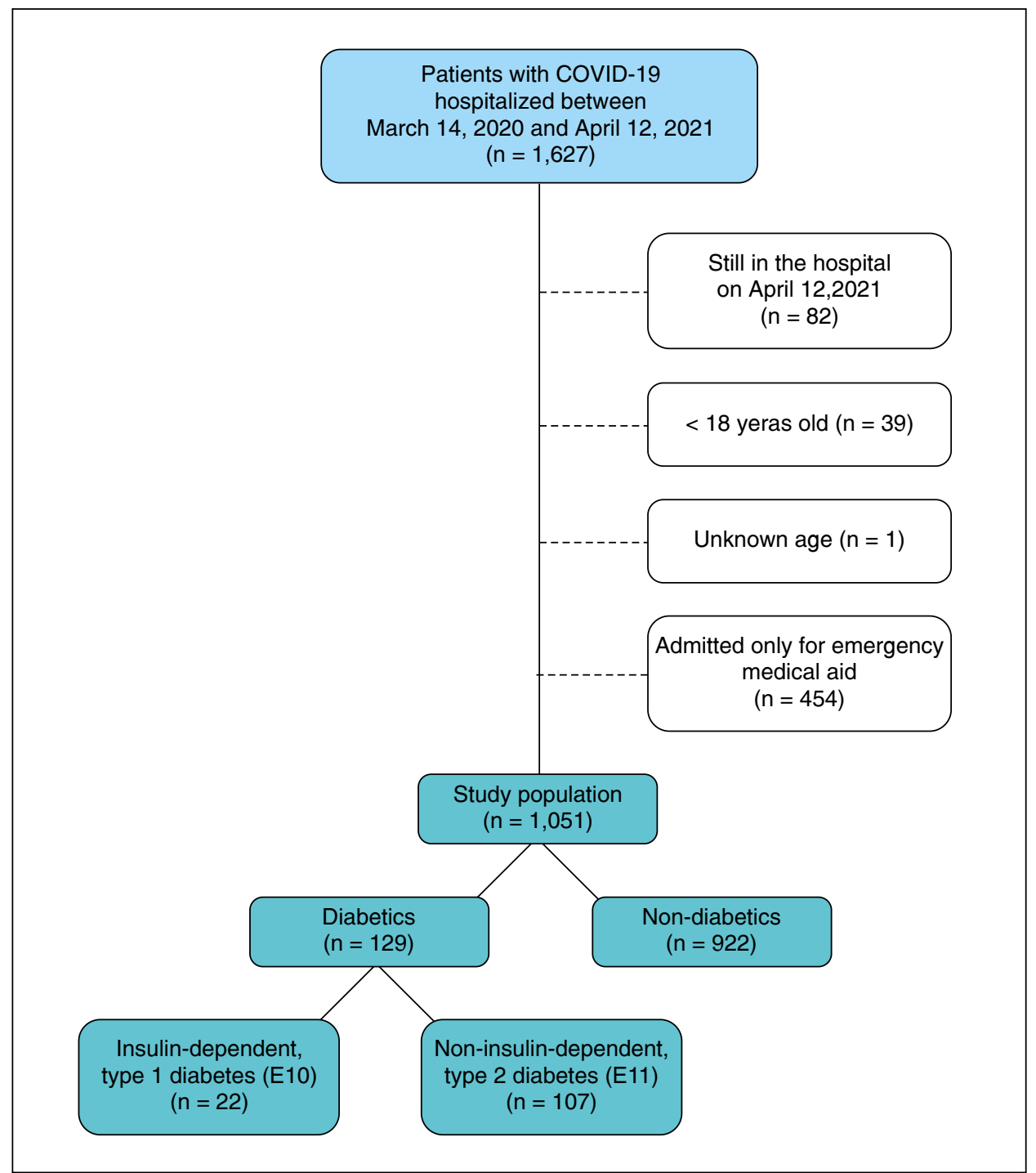

Figure 1. A flowchart of patients included in the analysis

are being proposed including immune response impairment [5]. The role of angiotensin-converting enzyme 2 is also being discussed [6]. The pro-coagulative and pro-inflammatory state exhibited by diabetic patients may be another reason for poor clinical outcome in this population [1]. However, the exact mechanisms are yet to be confirmed.

The matter of SARS-CoV-2 infection with concomitant diabetes still requires better understanding and intensive research to determine whether this combination constitutes a deadly duo. This paper aimed to analyse the relation of COVID-19 and diabetes, considering diabetes types 1 and 2, in respect of their influence on hospitalization's length and outcome with special regard to the issue of mortality. Prevalence of diabetes in COVID-19 positive patients was another objective of the study.

\section{Materials and methods}

The data acquired from the database of Antoni Jurasz University Hospital No. 1. in Bydgoszcz were analysed. The analysis included 1,051 adult COVID-19 positive patients that were hospitalized between March 14, 2020, and April 12, 2021. Patients who were still in the hospital at the time of data acquisition (April 12, 2021) were not included in the analysis. Patients under the age of 18 or unknown age, as well as those admitted to the hospital only for emergency medical aid were also excluded. A flowchart of the study is presented in Figure 1. The collected data included:

- history of diabetes defined by the 10th revision of the International Classification of Diseases (ICD-10) as insulin-dependent or type 1 diabetes mellitus (E10) and non-insulin-dependent or type 2 diabetes mellitus (E11); 


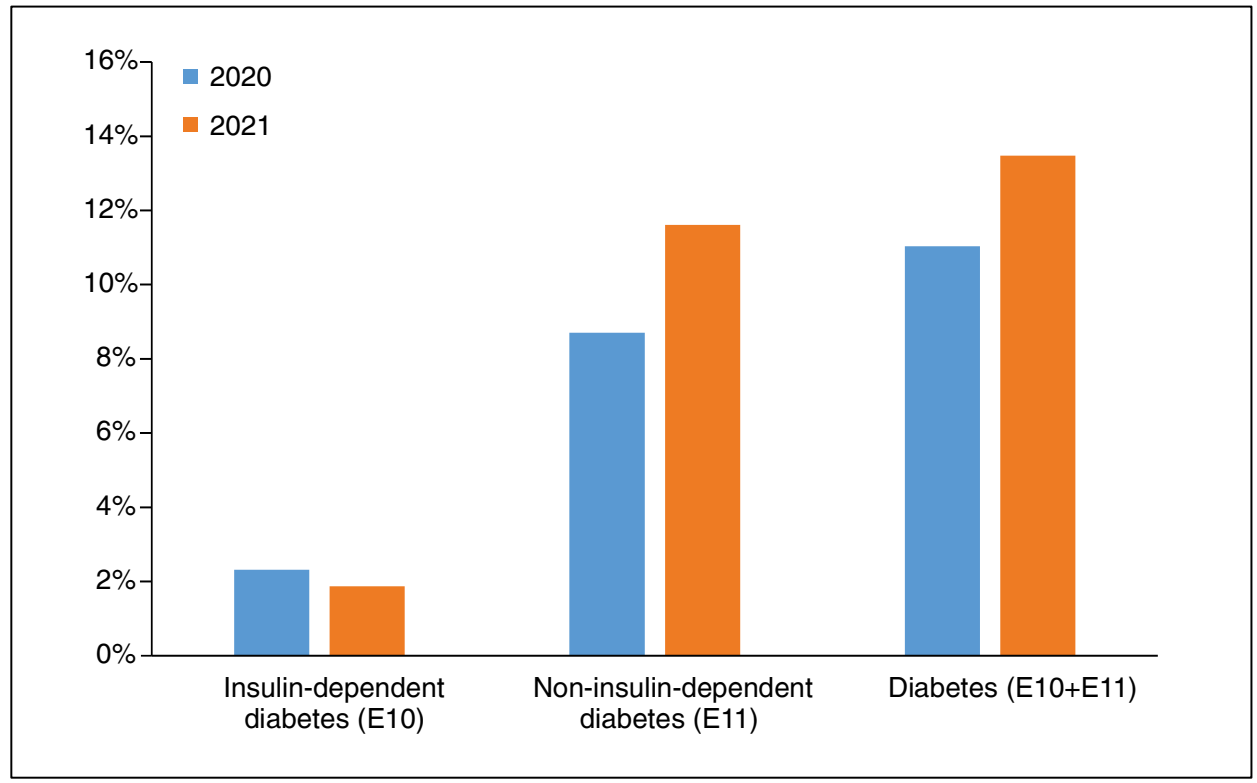

Figure 2. A comparison of diabetes occurrence in the study population between the years 2020 and 2021

Table 1. Hospitalization outcome according to the presence of diabetes and its types

\begin{tabular}{|c|c|c|c|c|c|}
\hline \multirow[b]{2}{*}{ Insulin-dependent diabetes (E10) } & \multicolumn{2}{|c|}{$\begin{array}{c}\text { Discharge } \\
(n=920)\end{array}$} & \multicolumn{2}{|c|}{$\begin{array}{c}\text { Death } \\
(n=131)\end{array}$} & \multirow{2}{*}{$\begin{array}{c}\text { p } \\
0.0720\end{array}$} \\
\hline & $\mathrm{n}$ & 16 & $\mathrm{n}$ & 6 & \\
\hline & $\%$ & $1.74 \%$ & $\%$ & $4.58 \%$ & \\
\hline \multirow[t]{2}{*}{ Non-insulin-dependent diabetes (E11) } & $\mathrm{n}$ & 91 & $\mathrm{n}$ & 16 & 0.4279 \\
\hline & $\%$ & $9.89 \%$ & $\%$ & $12.21 \%$ & \\
\hline \multirow[t]{2}{*}{ Diabetes $(\mathrm{E} 10+\mathrm{E} 11)$} & $\mathrm{n}$ & 107 & $\mathrm{n}$ & 22 & 0.1053 \\
\hline & $\%$ & $11.63 \%$ & $\%$ & $16.79 \%$ & \\
\hline
\end{tabular}

- hospitalization outcome: discharge or death;

- length of hospital stay.

The statistical analysis was carried out using the Statistica 13.0 package (TIBCO Software Inc, California, USA). Continuous variables were presented as means with standard deviations and medians with interquartile ranges. The Shapiro-Wilk test demonstrated the non-normal distribution of the investigated data. Therefore, for comparison of continuous variables between subgroups nonparametric Mann-Whitney unpaired rank-sum test was used. Categorical variables were expressed as the number and the percentage. Categorical variables were compared using the $\chi 2$ test. Results were considered significant at $p<0.05$.

\section{Results}

Of the 1,051 patients included in the analysis, $22(2.09 \%)$ had insulin-dependent diabetes (E10) and $107(10.18 \%)$ had non-insulin-dependent diabetes (E11), totaling to $129(12.27 \%)$ cases of diabetes (E10 + E11) in the study population (Fig. 1). The comparison of 2020 and 2021 periods regarding the occurrence of diabetes did not yield statistically significant differences $(p=0.2242)$, meaning the prevalence of diabetes did not differ between the patients hospitalized in $2020(n=517)$ and $2021(n=534)$ as it was found to be $11.03 \%$ and $13.48 \%$, respectively. In a separate analysis of insulin-dependent and non-insulin-dependent subgroups, statistically significant differences were not found either. The comparison of diabetes occurrence in 2020 and 2021 among the study population is presented in Figure 2.

An analysis of the hospitalization outcome in COVID-19 positive patients indicated that 920 (87.54\%) of the patients have been discharged and 131 (12.46\%) have died. The data were evaluated regarding diabetes type subgroups as shown in Table 1. In the case of insulin-dependent diabetes (E10) a pronounced, yet not deemed statistically significant, difference in its outcome contribution was noted as it constituted $4.58 \%$ of deaths and only $1.74 \%$ of discharges. 


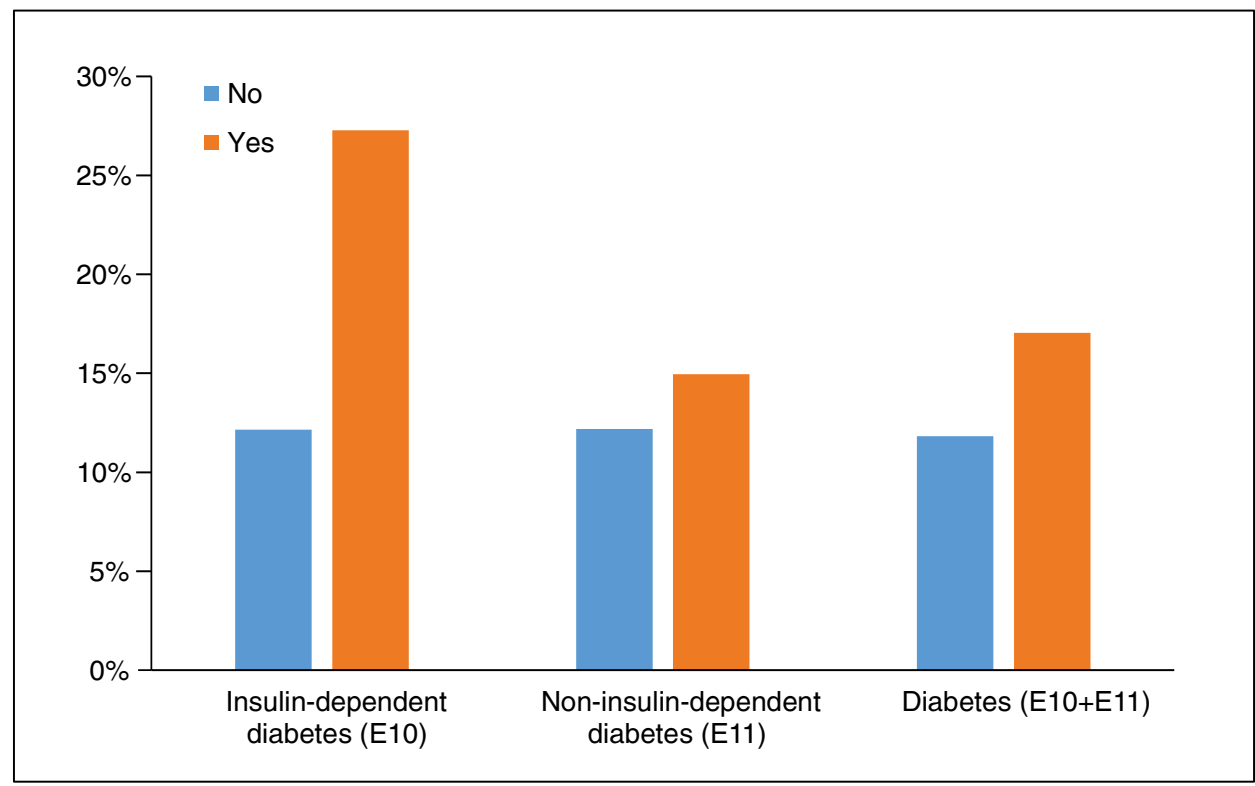

Figure 3. Mortality rates according to the presence of diabetes and its types

Table 2. Length of hospital stay according to the presence of diabetes and its types

\begin{tabular}{|c|c|c|c|c|c|c|c|}
\hline & \multicolumn{6}{|c|}{ Length of hospital stay [days] } & \multirow[t]{2}{*}{$\mathbf{p}$} \\
\hline & $\mathbf{n}$ & Mean & SD & Median & Q1 & Q3 & \\
\hline \multicolumn{8}{|c|}{ Insulin-dependent diabetes (E10) } \\
\hline No & 904 & 14.63 & 25.22 & 11.50 & 5.00 & 16.00 & 0.9481 \\
\hline Yes & 16 & 13.31 & 10.73 & 12.00 & 9.50 & 16.00 & \\
\hline \multicolumn{8}{|c|}{ Non-insulin-dependent diabetes (E11) } \\
\hline No & 829 & 14.42 & 23.77 & 11.00 & 5.00 & 16.00 & 0.2937 \\
\hline Yes & 91 & 16.35 & 34.63 & 12.00 & 7.00 & 16.00 & \\
\hline \multicolumn{8}{|c|}{ Diabetes $(\mathrm{E} 10$ + E11) } \\
\hline No & 813 & 14.44 & 23.96 & 11.00 & 5.00 & 16.00 & 0.3151 \\
\hline Yes & 107 & 15.90 & 32.18 & 12.00 & 7.00 & 16.00 & \\
\hline
\end{tabular}

A more detailed analysis of hospitalizations that ended in patient's death $(n=131)$ was performed to determine the mortality rates according to the presence of diabetes, which are shown in Figure 3. The mortality rates in the group of insulin-dependent diabetes (E10) patients were found to be the highest among all the analysed subgroups, namely $27.27 \%$ vs. $12.15 \%$ in the reaming study population $(p=0.0720)$.

The data on the length of hospital stay were acquired and analysed for the patients whose hospitalization ended in discharge $(n=920)$ as presented in Table 2. The average length of hospitalization for patients with any type of diabetes was determined to be 15.90 days vs. 14.44 days for the COVID-19 positive patients without diabetes $(p=0.3151)$. Considering specific types of diabetes separately, the patients with insulin-dependent and non-insulin-dependent diabetes on average stayed in the hospital for 13.31 and 16.35 days, respectively. No statistically significant differences were found between the groups and subgroups of patients with diabetes and the rest of the study population without it.

\section{Discussion}

The present study constitutes a much-needed attempt at analysing the relationship between COVID-19 and diabetes regarding hospitalization's length and outcome. The inclusion of type 1 diabetes patients into this analysis was found to be one of its strong points since most currently available studies only relate to type 2 diabetes. Other distinguishing features of 
the present study in the context of available publications include a longer analysis period and a relatively large number of patients for a single centre study.

The prevalence of diabetes among patients with COVID-19 has been analysed in several mostly single centre studies. Seiglie et al. [3] found that in March-April 2020 period at the Massachusetts General Hospital 178 out of 450 (39.6\%) COVID-19 patients had diabetes. It is noted that most of the diabetic patients had type 2 diabetes, with only two cases of type 1 diabetes. A onemonth-long study (February 26, 2020, to March 26, 2020) conducted by Akbariqomi et al. [7] in a hospital in Iran found that 1 in 4 COVID-19 patients had diabetes (148 of $595 ; 24.9 \%)$. In a study by Vargas-Vázquez et al. [8] based in a Mexican COVID-19 centre during the March-July period of $2020,50.2 \%$ of patients had type 2 diabetes (159 out of 317 ), with more than one-third of them being previously undiagnosed. However, such a high rate may have been a result of strict patient inclusion criteria as 600 patients were excluded due to e.g., lack of $\mathrm{HbA} 1 \mathrm{c}$ measurement on admission. On the other hand, Wang et al. [9] determined the prevalence of diabetes to be $1.7 \%$ as it was present in 126 out of 7,283 severe or critically ill COVID-19 positive patients in Wuhan hospitals as of February 25, 2020. Similarly, a low occurrence was reported by You et al. [10] in a study based on the National Health Insurance Service database in Korea in which between January and March of 2020 out of 5,473 analysed patients 495 (9.04\%) had type 2 diabetes. A wide variance in diabetes prevalence among COVID-19 patients presented in the aforementioned publications and the present paper could be a reflection of diverse populations from different geographic locations as well as the exclusion and inclusion criteria chosen for each of the studies. Notably, most of the available analyses either do not differentiate diabetes type or only consider type 2 diabetes.

This study results suggest that in the case of concomitant type 1 diabetes, COVID-19 positive patients may demonstrate higher mortality rates. The relation of SARS-CoV-2 infection and diabetes is currently being extensively researched with several studies aiming to establish the mortality rates in COVID-19 patients with diabetes versus those without. You et al. [10] determined the above-mentioned rates to be $5.7 \%$ and $1.1 \%$ ( $p<0.0001)$, respectively, with type 2 diabetes patients presenting with poorer clinical outcomes e.g., higher incidence of pneumonia. In a study by Seiglie et al. [3] the diabetic group was characterized by a higher proportion of mechanical ventilation and admissions to the intensive care unit. The mortality rates among diabetic COVID-19 patients at 14 days were determined to be $15.9 \%$ vs. $7.9 \%$ ( $p=0.009$ ) in non-diabetic patients. Based on those results diabetes has been hailed as a risk factor for poor early outcome in COVID-19 pa- tients. A longer observation period of 30 days yielded comparable results and conclusions since the mortality was significantly higher in patients with type 2 diabetes than in the group without (13.6\% vs $8.7 \%$; $p<0.001$ ) as reported by Sonmez et al. [11]. Moreover, pre-diabetes and undiagnosed type 2 diabetes were also found to be risk factors for severe SARS-CoV-2 infection [8].

The aforementioned studies pertain to either diabetes in general or type 2 diabetes only. The data on type 1 diabetes specifically are far more limited. A UK nationwide study by Ruan et al. [12], which aimed to assess the clinical characteristics and risk factors of adults with both COVID-19 and type 1 diabetes, reported 53 deaths among 194 analysed patients, constituting a mortality rate of $27.32 \%$. This result is very much consistent with the rate of $27.27 \%$ determined in this study. Yet, in this analysis, the diabetic group was not compared to a non-diabetic one. Two separate whole-population studies, one conducted in Scotland [13], one in England [14], found that both diabetes type 1 and 2, when adjusted for characteristics such as age, were independently associated with increased risk of fatal COVID-19 compared to the non-diabetic population (Scotland: $\mathrm{OR}=2.396$ in type $1, \mathrm{OR}=1.369$ in type 2; England: $\mathrm{OR}=3.51$ in type $1, \mathrm{OR}=2.03$ in type 2). Interestingly, the multicentre French CORONADO study [15] reported that the primary outcome of tracheal intubation for mechanical ventilation and/or death within 7 days of admission was not affected by diabetes type, meaning there were no differences between diabetes types 1 and 2 in respect of COVID-19 outcome. However, out of 1,317 participants, the study included only 39 patients with insulin-dependent diabetes.

According to these results, the length of hospitalization was not affected by the presence of diabetes. Contrastingly, in a nationwide study based on the Turkish Ministry of Health database, Sonmez et al. [11] reported a significantly higher rate of prolonged hospitalization, defined as a hospital stay of more than 8 days, among 9,213 patients with type 2 diabetes. The median length of hospital stay in the entire study population of 18,426 patients hospitalized for COVID-19 was 8 days. However, the patients with type 1 diabetes were excluded from this analysis. Several single centre studies are consistent with those results. Al-Salameh et al. [16] found the median length of stay to be longer in the case of COVID-19 patients with diabetes $(17.1 \pm 11.7$ days $)$ than in those without (13.5 \pm 9.1 days). In this study the group of diabetic patients composed of type 2 diabetes in $96.5 \%$. Alkundi et al. [17] reported comparable results with the length of hospitalization of diabetic and non-diabetic COVID-19 patients of $14.4 \pm 9.6$ and $9.8 \pm 17.1$ days, respectively. Here the structure of the study population regarding the type of diabetes was 
similar to the one presented in this study as $12.65 \%$ of diabetic patients had type 1 diabetes. Most available publications report a significantly longer time of a hospital stay among diabetic versus non-diabetic COVID-19 patients.

Some of the limitations of this study include not considering other comorbidities and patients' characteristics such as age that could have impacted the length of hospital stay and its outcome.

\section{Conclusions}

- COVID-19 and type 1 diabetes may constitute a deadly duo characterized by higher mortality rates than in the non-diabetic population.

- Further studies that include patients with type 1 diabetes are needed to better understand the impact of diabetes and COVID-19 on mortality and hospitalization's length.

\section{Statement of competing interests: The authors report no competing interests.}

List of abbreviations: COVID-19 - coronavirus disease 2019

SARS-CoV-2 - severe acute respiratory syndrome coronavirus 2

\section{References}

1. Apicella M, Campopiano MC, Mantuano M, et al. COVID-19 in people with diabetes: understanding the reasons for worse outcomes. Lancet Diabetes Endocrinol. 2020; 8(9): 782-792, doi: 10.1016/S22138587(20)30238-2, indexed in Pubmed: 32687793

2. Saeedi P, Salpea P, Karuranga S, et al. IDF Diabetes Atlas Committee Global and regional diabetes prevalence estimates for 2019 and projections for 2030 and 2045: Results from the International Diabetes Federation Diabetes Atlas, 9 edition. Diabetes Res Clin Pract. 2019; 157: 107843, doi: 10.1016/j.diabres.2019.107843, indexed in Pubmed: 31518657.

3. Seiglie J, Platt J, Cromer SJ, et al. Diabetes as a risk factor for poor early outcomes in patients hospitalized with COVID-19. Diabetes
Care. 2020; 43(12): 2938-2944, doi: 10.2337/dc20-1506, indexed in Pubmed: 32847827.

4. Knapp S. Diabetes and infection: is there a link?--A mini-review. Gerontology. 2013; 59(2): 99-104, doi: 10.1159/000345107, indexed in Pubmed: 23182884

5. Hussain A, Bhowmik B, do Vale Moreira NC. COVID-19 and diabetes: Knowledge in progress. Diabetes Res Clin Pract. 2020; 162: 108142 , doi: 10.1016/j.diabres.2020.108142, indexed in Pubmed: 32278764

6. Ma RCW, Holt RIG. COVID-19 and diabetes. Diabet Med. 2020; 37(5): 723-725, doi: 10.1111/dme.14300, indexed in Pubmed: 32242990.

7. Akbariqomi M, Hosseini MS, Rashidiani $\mathrm{J}$, et al. Clinical characteristics and outcome of hospitalized COVID-19 patients with diabetes: A single-center, retrospective study in Iran. Diabetes Res Clin Pract. 2020; 169: 108467, doi: 10.1016/j.diabres.2020.108467, indexed in Pubmed: 32979419

8. Vargas-Vázquez A, Bello-Chavolla OY, Ortiz-Brizuela E, et al. Impact of undiagnosed type 2 diabetes and pre-diabetes on severity and mortality for SARS-CoV-2 infection. BMJ Open Diabetes Res Care. 2021; 9(1), doi: 10.1136/bmjdrc-2020-002026, indexed in Pubmed: 33593750.

9. Wang F, Cao J, Yu Y, et al. Epidemiological characteristics of patients with severe COVID-19 infection in Wuhan, China: evidence from a retrospective observational study. Int J Epidemiol. 2021; 49(6): 1940-1950, doi: 10.1093/ije/dyaa180, indexed in Pubmed: 33150437

10. You JiH, Lee SAh, Chun SY, et al. Clinical outcomes of COVID-19 patients with type 2 diabetes: A population-based study in Korea. Endocrinol Metab (Seoul). 2020; 35(4): 901-908, doi: 10.3803/EnM.2020.787, indexed in Pubmed: 33297603.

11. Sonmez A, Demirci I, Haymana C, et al. Clinical characteristics and outcomes of COVID-19 in patients with type 2 diabetes in Turkey: A nationwide study (TurCoviDia). J Diabetes. 2021; 13(7): 585-595, doi: 10.1111/1753-0407.13171, indexed in Pubmed: 33655669.

12. Ruan $Y$, Ryder REJ, De P, et al. ABCD Covid-19 audit group. A UK nationwide study of people with type 1 diabetes admitted to hospital with COVID-19 infection. Diabetologia. 2021 [Epub ahead of print], doi: 10.1007/s00125-021-05463-x, indexed in Pubmed: 33966090.

13. McGurnaghan SJ, Weir A, Bishop J, et al. Public Health Scotland COVID-19 Health Protection Study Group, Scottish Diabetes Research Network Epidemiology Group. Risks of and risk factors for COVID-19 disease in people with diabetes: a cohort study of the total population of Scotland. Lancet Diabetes Endocrinol. 2021; 9(2): 82-93, doi: 10.1016/S2213-8587(20)30405-8, indexed in Pubmed: 33357491.

14. Barron E, Bakhai C, Kar P, et al. Associations of type 1 and type 2 diabetes with COVID-19-related mortality in England: a whole-population study. Lancet Diabetes Endocrinol. 2020; 8(10): 813-822, doi: 10.1016/S2213-8587(20)30272-2, indexed in Pubmed: 32798472

15. Cariou B, Hadjadj S, Wargny M, et al. CORONADO investigators. Phenotypic characteristics and prognosis of inpatients with COVID-19 and diabetes: the CORONADO study. Diabetologia. 2020; 63(8): 1500-1515, doi: 10.1007/s00125-020-05180-x, indexed in Pubmed: 32472191.

16. Al-Salameh A, Lanoix JP, Bennis Y, et al. Characteristics and outcomes of COVID-19 in hospitalized patients with and without diabetes. Diabetes Metab Res Rev. 2021; 37(3): e3388, doi: 10.1002/dmrr.3388, indexed in Pubmed: 32683744

17. Alkundi A, Mahmoud I, Musa A, et al. Clinical characteristics and outcomes of COVID-19 hospitalized patients with diabetes in the United Kingdom: A retrospective single centre study. Diabetes Res Clin Pract. 2020; 165: 108263, doi: 10.1016/j.diabres.2020.108263, indexed in Pubmed: 32531325. 